\title{
Emerging Technologies (Game-Based Learning and Virtual Reality) for Enhancing Learner Engagement in Humanitarian Contexts
}

\author{
Atish Gonsalves, Kabir David, Melisa Zelaya \\ Leadership Academy, Quicksand, Humanitarian Leadership Academy
}

\begin{abstract}
The research was framed around the hypothesis that emerging, innovative, technologyenabled learning formats, while bringing additional delivery and access challenges, compared to traditional eLearning formats, could lead to higher user engagement and better learning outcomes. Formats were selected that have been successfully tested in other fields. The objective of using innovative, technology-enabled learning formats was to incorporate more human-to-human engagement and empathy building through immersive game-based learning and virtual reality (VR).
\end{abstract}

Keywords: Education and Research Management, Special Education. 


\section{$2^{\text {nd }}$ International Conference on Research in TEACHING and EDUCATION}

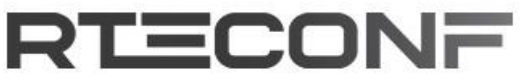

6-8 March, 2020 BUDAPEST,HUNGARY

\section{Introduction}

The design of engaging, effective and scalable technology-enhanced learning experiences is complex and requires a learner-centric approach, grounded in evidence-based pedagogies. The Humanitarian Leadership Academy (Academy) has developed a number of learning technologies with a design-based approach which allows for multiple iterations of the experience based on constant user feedback.

The research model is supported by the use of human-centred design (HCD) for the development of the learning experiences. HCD draws from design theories in which the needs, wants and limitations of the end user of a product are the focus of each stage of the design process. By involving the user at each phase of the development process, the functionality and relevance of the product is ensured. (Garreta-Domingo \& Mor, 2006).

The learning experiences developed are all relevant for the humanitarian sector, as the core objective of the Academy is to deliver relevant and accessible humanitarian learning, and it is important to acknowledge the particular nuances and challenges of the field.

In recent years, there has been an increase in the interest in humanitarian innovations, as a way of changing the traditional methods of humanitarian aid delivery and assistance. However, a significant amount of the existing approaches to humanitarian innovation have followed a top-down approach. While this approach is valuable and offers new solutions that can improve organizational responses, it is not the only way to approach humanitarian innovation. (Blooms \& Betts, 2013).

Another way to approach humanitarian innovation fosters and builds from the existing capabilities of local communities. This bottom-up approach is preferable as the solutions that come from it are more likely to be accepted by local communities and are more likely to be sustainable.

Despite the welcome interest and investment in operational innovation seen in the humanitarian sector over recent years, more needs to be done to promote these bottom-up approaches in innovations in learning. To address this need, the Academy's initial approaches to innovation leverage not just technology, but new tools and methodologies to solve problems in locally relevant and creative ways.

\section{Research objectives}

The research was framed around the hypothesis that emerging, innovative, technologyenabled learning formats, while bringing additional delivery and access challenges, compared to traditional online learning formats, could lead to higher user engagement and better learning outcomes. Formats were selected that have been successfully tested in other fields. The objective of using innovative, technology-enabled learning formats was to incorporate more human-to-human engagement and empathy building through immersive virtual reality and game-based learning. 


\section{$2^{\text {nd }}$ International Conference on Research in TEACHING and EDUCATION}

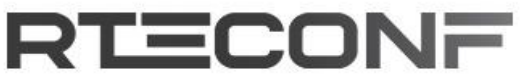

6-8 March, 2020 BUDAPEST,HUNGARY

With the longstanding focus on learning and knowledge, the Academy attempts to answer the question:

"How do we co-create and share learning that is engaging, scalable and impactful to build strong, resilient communities and save lives?"

In the context of humanitarian work, the targeted community groups are rarely involved in the design of the programmes (Proudlock and Ramalingam 2008). These lack of integration with the communities may result in a lack of sensitivity for local practices and existing structures. The innovation outputs from the Academy put at the centre of the learning process the endeavour to enable others to create and share their own experiences, thus becoming active creators of learning rather than being passive consumers of knowledge.

To this end, the Academy partnered with Quicksand (India) to augment its learning offering by iteratively co-creating, testing and evaluating a number of innovative learning formats and technologies to address the increasingly complex needs of the humanitarian sector.

\section{Project background and methodology}

In recent years, there has been an increased interest and availability of literature focused on technology-enhanced learning environments. These types of instructional systems in which learners acquire skills and knowledge are based in the inclusion or learning support tools and technological resources. (Aleven, Stahl, Schworm, Fischer, \& Wallace, 2003).

Building on this trend, the Academy has implemented the human-centred design (HCD) approach to explore and research technologically-enhanced learning systems. The designresearch paradigm advances synergic relationships among researchers and designers and adds values through the active involvement of researchers in learning procedures through "scientific processes of discovery, exploration, confirmation, and dissemination" (Kelly, 2003, p. 3).

The design-based research is defined as a flexible methodology with the primary objective to improve educational practices through iterative analysis, design, development, and implementation based in a collaboration process in real-world settings, its five main characteristics are the following: (a) pragmatic; (b) grounded; (c) interactive, iterative, and flexible; (d) integrative; and (e) contextual. (Wang \& Hannafin, 2005).

The project introduced two emerging formats of learning content - game-based learning and immersive media (using 360 films and interactive VR experiences). Content was then developed to use the strengths of each medium, each geared towards a unique set of learning outcomes. 


\section{$2^{\text {nd }}$ International Conference on Research in \\ TEACHING and EDUCATION}

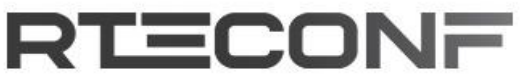

6-8 March, 2020

BUDAPEST,HUNGARY

\section{A. Game-based Learning}

Computer games evoke mixed reactions, on the one hand there are many who argue that there's no positive outcome from the amount of time spent in virtual worlds, on the other hand, many games have been seen as instructive and engaging. Regardless of how they are perceived, the fact is that games have solidified a place in the markets and in popular culture. In addition to their commercial success, games have captured the attention of educators, there are several reasons for this interest.

First, in recent times there has been a shift in the field of learning from a traditional, didactic model of instruction, to a more dynamic type of learning which emphasizes on the active role of the learner. (Garris, 2002). A second reason is that some empirical evidence suggests that games can be effective tools for enhancing learning and to advance the understanding of complex subject matters. (Cordova \& Lepper, 1996). Thirdly, there is an interest in the intensity of the involvement and engagement that games can provide. Achieving the aforementioned motivational properties of games to enhance learning and accomplish learning objectives would mean that both objectives have been successfully met.

The key goal of game-based learning experiences was to scale human engagement in learning while achieving learning objectives, via role-playing, scenarios, peer-to-peer learning and simulation exercises. The content for the games was co-created with local partners using HCD approaches on the Gamoteca tool (gamoteca.com), and were rolled out via the Academy's learning platform, Kaya (kayaconnect.org). The learning games included coaching and mentoring, empathy building through disaster scenarios for volunteers, implicit gender bias, design thinking and onboarding for new teams.

\section{B. Immersive Films}

Low-tech, low-cost 360 virtual reality (VR) experiences were identified as a content format for immersing the user in new environments and building empathy with people affected by disasters. Its key characteristic was the ease of fully immersing the user into a new environment, scalability via social media platforms like YouTube 360 and Facebook and ease of integration into existing learning pathways on Kaya. In addition to VR headsets like Oculus, smartphones combined with VR goggles, e.g. Google Cardboard, also offer a low-cost way of accessing VR content. Interactive VR experiences developed on Unity, e.g. Camp 2029, also allowed the user to interact with their virtual world, in this case a dystopian humanitarian refugee camp. 


\section{$2^{\text {nd }}$ International Conference on Research in \\ TEACHING and EDUCATION}

\section{Design Approach}

The themes of the outputs were determined in the following ways:

\section{A. Locally Inspired Co-Creation}

- Certain concepts were developed through co-creation workshops using design thinking with local partners (in Kenya, the Philippines, Bangladesh, Jordan and India), drawing on local knowledge, expertise and stories.

- Some of these outputs were further developed by Quicksand in close collaboration with the initial creators of the concept to ensure their authenticity was retained.

\section{B. Complementary Learning Content}

- Existing learning experiences that could be easily complemented by additional resources were identified, e.g. Coaching \& Mentoring and Volunteer Essentials courses on Kaya.

- New ideas and themes were identified based on specific learning gaps or opportunities.

\section{Development Process}

Quicksand reviewed the Academy's existing learning services, primarily its Kaya platform, to deepen its understanding of the ecosystem in which the new content would be integrated. This built the foundation that would inform the development and positioning of the project's outputs. This was especially important for the outputs that would complement existing learning resources.

New learning outputs were created following a larger process that was modified according to the constraints of each case:

- Identifying the theme and key learning objectives based on user-centred design principles.

- Establishing a broad flow of what the experience would look like.

- Building a storyboard of the same flow.

- Creating a prototype of the product.

- Testing with a sample user set.

- Iterative development of the product based on user feedback. 


\section{$2^{\text {nd }}$ International Conference on Research in \\ TEACHING and EDUCATION}

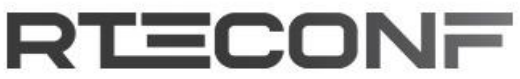

6-8 March, 2020

BUDAPEST,HUNGARY

\section{Qualitative Testing and findings}

Qualitative testing of a selected set of the new learning resources outputs was conducted with roughly 30 participants. Each session followed a fixed process:

1. Introduction: Each participant was first introduced to the project and its scope in order to establish a clear understanding of the purpose of the testing session. Basic characteristics of the participant were also documented (age, occupation etc).

2. Experiencing the output: Participants were left to experience the output for themselves. In the case of the immersive films, they were given a VR headset to watch the experience, if they felt comfortable doing so. In the case of group-based games, they were either paired with another participant or with a member of the testing team.

3. Feedback: Before any words were exchanged, they were asked to list out their main impressions of the experience on post-its. This was done to ensure that each participant's initial feedback was authentic and not biased by any prompts or direct questions.

4. Discussion: An open discussion that built on the participant's feedback and a preidentified set of conversation topics was held with all the participants and moderated by the testing team.

5. Survey: The closing activity of the session was the filling of an online survey that helped capture key parameters in a quantitative manner.

\section{A. Game-based learning experiences}

The games tested spanned different formats and themes - from practicing coaching skills, to a role-playing journey in volunteering that served as an introduction to the world of humanitarian work.

\section{- Engagement}

Overall, users found themselves to be more actively engaged while using the outputs when compared to more traditional learning formats. This included a higher level of attentiveness, and a higher level of enthusiasm with regards to the subject matter.

Based on participant responses, drivers of higher engagement were seen to be role and narrative-based game journeys. The deeper the level of immersiveness, augmented by elements like decision-making and role-playing, the deeper the engagement with the learning. 


\section{$2^{\text {nd }}$ International Conference on Research in \\ TEACHING and EDUCATION}

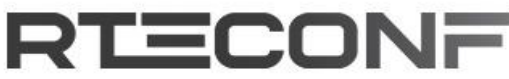

6-8 March, 2020

BUDAPEST,HUNGARY

\section{- Group-based learning is a key driver of engagement}

Games that involved more than one player were seen to facilitate increased engagement with the learning material. Interacting with another person within the experience led to higher attentiveness in most learners, while also giving them a sense of being part of a larger exercise.

"I feel this is a great way to teach a skill that people often find boring. When learning doesn't have a two-way engagement, the receiver often feels she/he is not contributing anything to the learning process. Also, in this game you get to engage with mentors across the globe."

A participant after playing the "Coaching and Mentoring" game.

\section{- Interactivity provides agency}

Exercises and activities within the games - both individual as well as with other learners - was found to give learners a sense of agency and ownership over the journey, allowing them to define their own learnings and key takeaways from the experience. In most cases, these still aligned with the broader learning objectives established for each game.

"This is definitely a fun and interactive way to learn. I feel the visual and audio element in the game helped in relating to the situation at hand. This could be a great tool to use in our work, too, when we train the youth on relationship building, so definitely see myself using this in my training. "

A participant after playing the "Coaching and Mentoring" game.

"It was a lot more interactive than simply reading or watching something and gives a good perspective into both sides [of humanitarian response]."

A participant after playing the "The Flood: Multiplayer" game

\section{- Immersiveness}

Given that most of the outputs tested were narrative-driven experiences, the extent of their immersiveness was a key factor to gauge through the testing process. The games' immersiveness was seen to depend on how convincing the narrative was, as well as the integration of activities and decision-making points.

\section{- Role-playing helps heighten immersion}

Role-playing allowed learners to empathise and understand another person's context. With sufficient context, learners were seen to relate better and invest in their characters and corresponding activities. 


\section{$2^{\text {nd }}$ International Conference on Research in \\ TEACHING and EDUCATION}

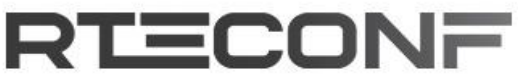

6-8 March, 2020

BUDAPEST,HUNGARY

"The game made me think about being in someone else's shoes and what their background story is."

A participant after playing the "The Flood: multiplayer" game

\section{- The more compelling the narrative, the deeper the learning}

A believable, nuanced narrative helps draw the learner in further into the experience, allowing for more impactful learning.

"Having a game like this adds a little more excitement to a learning experience. The point where I had to make a decision about whether (as a person affected by a flood) I should be with two of my children or go searching for my third was very powerful, it gave me a very concrete idea of what it could be like to be in such a situation."

A participant after playing the "The Flood: Multiplayer" game

\section{- Alignment with Learning Objectives}

The identified learning objectives were qualitative in nature, rather than easily measurable objectives. The learning objectives and included: practical coaching skills, empathy, and decision making. Data was collected through conversations and group discussions at the end of the participants' learning journey.

"Yes, it showed me what goes into volunteering in a time of calamity or distress."

A participant after having played The Flood: Multiplayer, whose main learning objective was to serve as an introductory exercise to a larger course.

"It gave an insight an insight into something that can be so complex in such a simple way."

A participant after playing the "Coaching and Mentoring" game.

\section{B. Immersive Films}

Each film created was deployed using a different format in order to cover a variety of approaches. This spanned $360^{\circ}$ video and interactive virtual reality (VR). You Cannot Argue with a Flood was made with $360^{\circ}$ video and focused on the Marawi (Philippines) conflict and a natural disaster told from the eyes of a survivor who is now a volunteer. 


\section{$2^{\text {nd }}$ International Conference on Research in \\ TEACHING and EDUCATION}

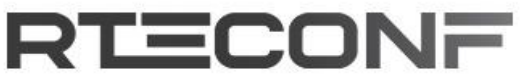

6-8 March, 2020

BUDAPEST,HUNGARY

Rohingya $V R$ presented interview accounts of different perspectives, refugees and local aid workers, in the Cox's Bazar refugee camps in Bangladesh, allowing the viewers to choose the interview they wanted to watch. Each of these experiences were distinct use cases to explore the potential offered by creating immersive environments through new technologies.

\section{- Engagement}

Initial user testing of each film pointed towards a clear capability to evoke emotion and empathy in viewers, indicative of the broader medium's scope to be leveraged as a tool for additional learning experiences.

"Before watching, I didn't think much of VR, but [it] helps you focus on the subject and free yourself from distractions. The experience stays with you for a bit."

"Gave the feeling of 'If she can do it, I can too. '"

"The gravity of the situation is established by the surroundings"

Participants after watching You Cannot Argue with a Flood

\section{- Immersiveness}

While the games' immersiveness was seen to depend on how convincing the narrative was, as well as the integration of activities and decision-making points, the nature of the $360^{\circ}$ film medium established a certain innate level of immersion, further augmented by the narrative and visual elements.

\section{- Emotion and empathy as complementary qualities}

Most cases reflected a direct relationship between the emotional factor of an experience and the level of empathy the learner experienced.

"It's a useful choice of medium as it immerses you in the story...helps you empathize with the narrator more. It brings alive the adage of 'being in someone else's shoes'. I could experience her pain more than in a normal video. Gives you the feeling of being in that place" 


\section{$2^{\text {nd }}$ International Conference on Research in \\ TEACHING and EDUCATION}

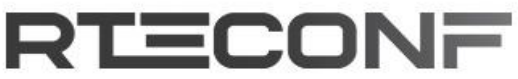

6-8 March, 2020

BUDAPEST,HUNGARY

"It definitely had a high empathy factor - I felt like I was really there."

Participants after watching the film You Cannot Argue with a Flood.

"These films make the viewer very emotional."

"It was good to get many perspectives and stories. The stories are personal and extremely emotional."

Participants after watching Rohingya Refugee Crisis

\section{- Alignment with Learning Objectives}

The primary learning objectives from the videos were empathy building and learner sensitisation to new contexts. These were met in most cases, however, the new nature of the medium brought up some considerations around how such experiences can be designed and implemented for first-time users.

The experiences seemed particularly useful while showing a new environment to the users. They pointed out that being capable of seeing what someone in a disaster goes through was eye-opening and allowed them to understand the context better. Users complimented the quality of the film scenes and highlighted that the clarity of the video increased the empathic capacities of the film.

"Very nicely shot - very powerful if one uses the [VR] headset"

Participant after watching You Cannot Argue with a Flood

\section{- Feeling Overwhelmed}

In the case of the immersive films tested, some users were seen to feel slightly overwhelmed by the nature of the medium. There was a need for the flow of the film to be slower, giving the viewer more time to absorb the environment the film created.

"It took me some time to get acquainted with the visuals as I was following the dialogue, everything was presented too quickly and I couldn't keep up."

Participant after watching You Cannot Argue with a Flood 


\section{$2^{\text {nd }}$ International Conference on Research in \\ TEACHING and EDUCATION}

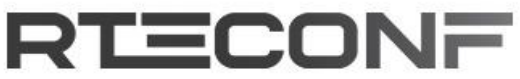

6-8 March, 2020

BUDAPEST,HUNGARY

\section{- Setting Expectations}

Participants typically weren't fully aware of what they were about to experience. Even after a briefing prior to the experience, some reactions of the users pointed towards a need to effectively explain the intent and nature of the experience - either within the concerned piece or outside of it.

\section{"I felt some parts of the film were staged."}

Participant after watching You Cannot Argue with a Flood

\section{Conclusions:}

The complex competencies required in the humanitarian sector, particularly in the areas of empathy building, prompted the creation of learning resources leveraging innovative formats and technologies, whose efficacy was tested through qualitative and quantitative evaluation methods.

In the context of existing and emerging complexities of humanitarian crises, there are crucial skills the humanitarian sector requires in order to ensure effective and appropriate delivery of aid and assistance.

The people-centred and urgent nature of humanitarian work calls for competencies such as adaptability, empathy, and quick decision making. Such capabilities are hard to bring to life through traditional learning formats due to their prescriptive, often theoretical approaches to learning.

Newer, innovative formats such as gamified learning and immersive technologies allow for the involvement of the user in the process of learning in a manner that previous approaches do not, including in its design process. In light of these hypotheses, innovative learning resources were created in the form of gamified experiences, immersive and interactive films.

These resources were then tested based on parameters such as the alignment of outcomes to desired learning goals, the relevance of the content, and the effectiveness of the medium, among others.

Findings from this testing and evaluation reflected a positive response and a resonance with the outlined learning objective, with each user responding to the outputs in a unique manner, demonstrating a deeper engagement with the subject matter. 


\section{$2^{\text {nd }}$ International Conference on Research in \\ TEACHING and EDUCATION}

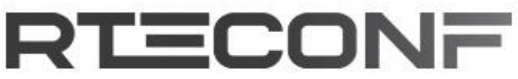

6-8 March, 2020

BUDAPEST,HUNGARY

\section{References}

Aleven, V., Stahl, E., Schworm, S., Fischer, F., \& Wallace, R. (2003). Help seeking and help design in interactive learning environments. Review of Educational Research, 73(3), 277-320

Bloom, L. \& Betts, A. (2013). The two worlds of humanitarian innovation. Working papers series No. 94. Refugee Studies Centre, University of Oxford.

Cordova, D. I., \& Lepper, M. R. (1996). Intrinsic motivation and the process of learning: Beneficial effects of contextualization, personalization, and choice. Journal of Educational Psychology, 88, 715-730.

Garreta-Domingo, M. \& Mor, E. (2006) User centered design in E-Learning environments: From usability to learner experience. Universitat Oberta de Catalunya Barcelona, Spain

Garris, R., Ahlers, R., \& Driskell, E. (2002) Games, Motivation, and Learning: A Research and Practice Model, Sage Publications.

Kelly, A. E. (2003). Research as design. Educational Researcher.

Kent, T. W., \& McNergney, R. F. (1999). Will technology really change education: From blackboard to Web. Thousand oaks, CA: Corwin Press

Proudlock, K. \& Ramalingam, B. 2008. re-thinking the impact of humanitarian aid: background paper for the 24th ALNAP Biannul. London: ALNAP.

Wang, F., \& Hannafin, M. J. (2005). Design-based research and technology-enhanced learning environments. Educational Technology Research and Development. 